\title{
Role of an Intensity-Transformation Function in Enhancement of Bone Scintigraphy Images
}

\author{
Anil Kumar Pandey ${ }^{1}$, Vishali Dhiman ${ }^{1}$, Akshima Sharma $^{1}$, Sreedharan Thankarajan ArunRaj ${ }^{1}$, Vivek Baghel ${ }^{1}$, \\ Chetan Patel ${ }^{1}$, Param Dev Sharma ${ }^{2}$, Chandra Sekhar Bal ${ }^{1}$, and Rakesh Kumar ${ }^{1}$ \\ ${ }^{1}$ Department of Nuclear Medicine, All India Institute of Medical Sciences, Ansari Nagar, New Delhi, India; and ${ }^{2}$ Department of \\ Computer Science, SGTB Khalsa College, University of Delhi, India
}

\begin{abstract}
Bone scintigraphy images might exceed the dynamic range (the ratio between the highest and the lowest displayable brightness) of the monitor. In such a case, a high-intensity area accompanied by loss of detail in other structures in the displayed image make the clinical interpretation challenging. We have investigated the role of an intensity-transformation (IT) function in enhancement of these types of images. Methods: Forty high-dynamic-range bone scintigraphy images were processed using an IT function. The IT function has 2 parameters: threshold and slope. With the threshold kept equal to the mean count of the image, the slope was varied from 1 to 20 . A software program developed in-house was used to process the images. Twenty output images corresponding to one input image were visually inspected by 2 experienced nuclear medicine physicians to select images of diagnostic quality, and from their selection was determined the standardized slope that produced the maximum number of diagnostic images. The 2 physicians also scored the quality of the input and output images (at the standardized slope) on a scale of 1-5. The Student $t$ test was used to determine the significance of differences in mean score between the input and output images at an a significance level of 0.05. Results: Application of the IT function with standardized parameters significantly improved the quality of highdynamic-range bone scintigraphy images $(P<0.001$, with $a=$ $0.05)$. A slope of 8 maximized the number of diagnostic images. Conclusion: The IT function has a significant role in enhancing high-dynamic-range bone scintigraphy images.
\end{abstract}

Key Words: high-dynamic-range bone scintigraphy image; intensity-transformation function; image enhancement

J Nucl Med Technol 2018; 46:274-279

DOI: 10.2967/jnmt.117.202929

B one is an active, constantly changing organ comprising inorganic calcium hydroxyapatite crystals and an organic matrix of collagen and blood vessels. Bone responds to injury and disease with increased turnover and attempts at self-repair.

\footnotetext{
Received Sep. 28, 2017; revision accepted Feb. 12, 2018.

For correspondence or reprints contact: Anil Kumar Pandey, Department of Nuclear Medicine, All India Institute of Medical Sciences, Ansari Nagar, New Delhi 110029, India.

E-mail: pandeyanilkumar@rediffmail.com

Published online Mar. 29, 2018.

COPYRIGHT (c) 2018 by the Society of Nuclear Medicine and Molecular Imaging.
}

This physiologic process can be imaged with ${ }^{99 \mathrm{~m}} \mathrm{Tc}$-methylene diphosphonate (MDP), which localizes to areas of bone formation. ${ }^{99 \mathrm{~m} T c-M D P}$ binds in the hydroxyapatite mineral component of the osseous matrix by chemisorption (1). Approximately $3 \mathrm{~h}$ after administration of ${ }^{99 \mathrm{~m} T c-M D P}$, wholebody digital images are acquired with a $\gamma$-camera. A scintigraphy image is a rectangular matrix of integer (intensity) values. Each element of the matrix corresponds to an image pixel with a depth of $8-16$ bits. The display controller-a graphic card or video card that controls the electronics of the display device-converts the intensity values into analog signals for display on a high-resolution cathode-ray-tube or liquid-crystal-display monitor for diagnostic interpretation (2).

The dynamic range of a monitor is defined as the ratio between the highest and lowest luminance (brightness) it can display. Generally, nuclear medicine images are low-count images with considerable statistical fluctuations; hence, a small difference in intensity is meaningless in such images. Therefore, a monitor that can accurately display 256 intensity values is most commonly used for nuclear medicine images (3). This number of values is also sufficient to produce a sequence of brightness levels perceived as continuous by the human observer.

However, there are situations in which the dynamic range of the input images exceeds the dynamic range of the monitor (e.g., extravasation, residual activity in the bladder, or uptake that is very high in a small area of the skeleton vs. the remainder). The monitor in such a case will not accurately display all the intensity levels present in the image. If the image has a single high-intensity area, the other structures in the image may not be visualized accurately, making the clinical interpretation difficult (Fig. 1).

To display these images in a way that would reveal more information of interest, some computer processing is required. In this study, we investigated an intensity-transformation (IT) function to enhance contrast in this category of bone scintigraphy images. The function increases contrast by compressing the lower and higher pixel-count values (intensity levels), thus increasing contrast simultaneously in both the darker and the brighter regions of the image (4). However, the threshold and slope parameters in the IT function need to be set manually to yield the best 


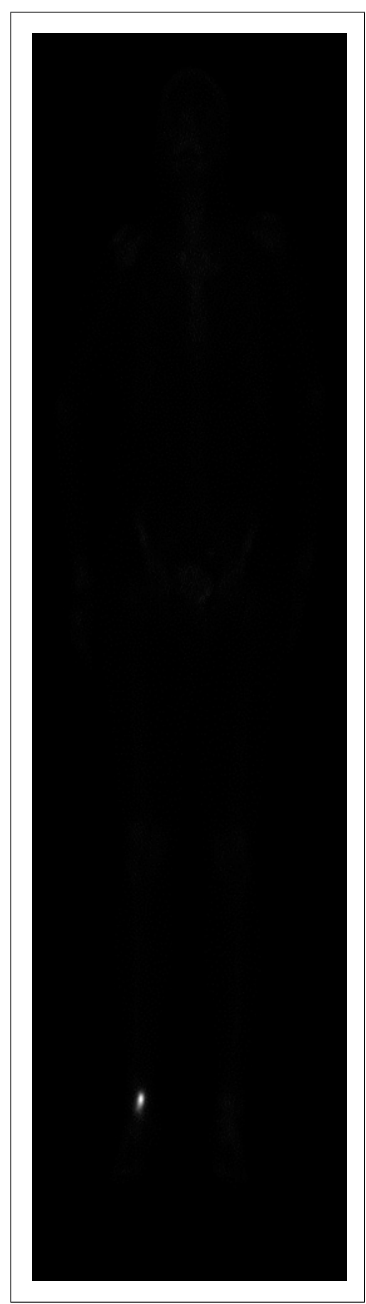

FIGURE 1. Appearance of whole-body 99mTc-MDP bone scintigraphy image when dynamic range of input image exceeds dynamic range of monitor.

results. In the present study, we attempted to standardize these input parameters.

\section{MATERIALS AND METHODS}

This retrospective study included the images of patients who had undergone bone scintigraphy in our facility between April 2015 and May 2016 for routine clinical indications. No ethical approval was required, as the study involved only the digital processing of anonymized images and included no direct contact with patients.

\section{Image Data Collection}

Forty unprocessed bone scintigraphy images whose visual appearance on the monitor was similar to the example shown in Figure 1 (i.e., with a high dynamic range) were exported in DICOM format. This set of images included 28 that were completely dark because of radiotracer extravasation, 4 that showed residual bladder activity, 5 that showed both extravasation and bladder activity, 2 that showed contamination and residual bladder activity, and 1 that showed catheter activity. Thus, this set of images included almost all the variations that can occur on bone scintigraphy images in routine practice.

The images were acquired on a dual-head $\gamma$-camera (Symbia E; Siemens Medical Solutions) equipped with a low-energy high- resolution collimator. Before administration of the ${ }^{99 \mathrm{~m}} \mathrm{Tc}-\mathrm{MDP}$, the patients were asked to drink at least 1-2 L of water and void their bladder frequently to reduce the radiation burden in the body. A 7- to 11-MBq dose of ${ }^{99 m}$ Tc-MDP per kilogram of body weight was administered intravenously. After an uptake period of 3-4 h, whole-body anterior and posterior images were acquired at a rate of approximately $1.66 \times 10^{-3} \mathrm{~m} / \mathrm{s}$ and a resolution of $256 \times 1,024$ pixels.

\section{IT Function}

The equation below describes the mathematic relationship between the input image pixel count and the output image pixel count:

$$
s=T(r)=\frac{1}{\left[1+\left(\frac{m}{r}\right)^{e}\right]},
$$

where $s$ is the pixel count of the output image, $r$ is the pixel count of the input image, $m$ is the threshold, and $e$ is the slope of the linear portion of the curve. The transform function, $T(r)$, compresses the input levels below and above the threshold into a narrow range of darker levels (lower pixel count) and brighter levels (higher pixel count) and thereby improves contrast (4). The characteristic curve of this function is illustrated in Figure 2, which shows that the counts above and below 127 are compressed at a slope of 1,4 , and 10, but each in a different fashion. At a fixed threshold, the value of the slope determines the shape of the curve and hence the quality of the output image.

\section{Image Processing}

A MATLAB (MathWorks) script was written to process the input images. All 40 images were processed at a fixed threshold (i.e., equaling the average intensity of the image) and with slope varied from 1 to 20 by an increment of 0.1 . In this way, a total of 8,000 output images (200 output images per input image) were produced. The team of 2 experienced nuclear medicine physicians found hardly any appreciable difference in image quality at an increment of 0.1 , but when the increment was increased to 1 , the team did see a difference. Therefore, the latter increment was applied to produce a total of 800 output images (20 output images per input image). The 2 observers reviewed the images

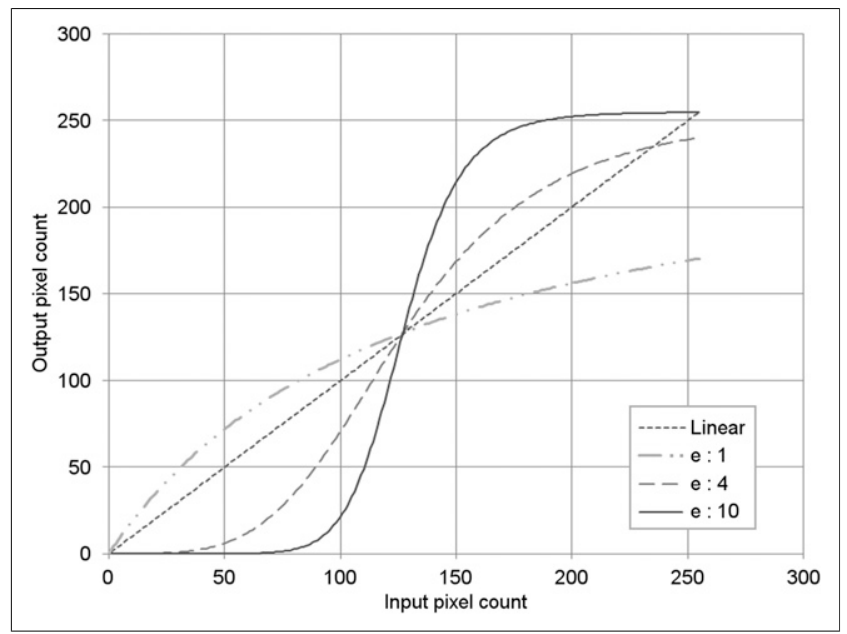

FIGURE 2. Characteristic curves for linear and IT function: linear transformation curve and IT curves with slopes (e) equaling 1, 4, and 10. In each case, threshold was 127. 


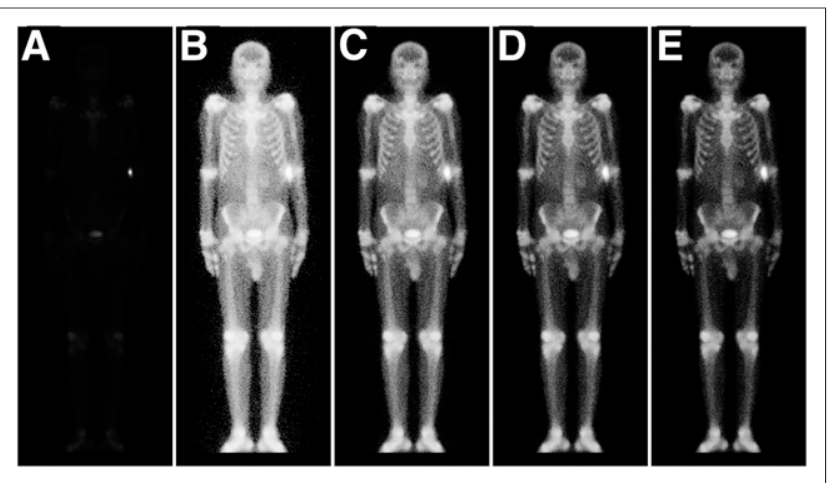

FIGURE 3. Whole-body ${ }^{99 m T c-M D P}$ bone scintigraphy images: input image $(A)$ and output images with slopes of 1-4 (B-E).

under the same ambient lighting conditions using a Hewlett-Packard Omni 120-1060 personal computer with a generic plug-and-play liquid-crystal-diode monitor that had a native resolution of $1,600 \times$ 900 pixels (high-definition-plus), a diagonal size of 20 in $(51 \mathrm{~cm})$, a response time of $5 \mathrm{~ms}$, and an image contrast ratio of 1,000:1. The experiments were performed on a personal computer having a Microsoft Windows 7 Home Basic 64-bit operating system, 2 GB of random-access memory, and an Intel Core i3-2120 central processing unit with a $3.30-\mathrm{GHz}$ processor.

\section{Visual Assessment of Image Quality}

Visual image quality was assessed in 2 steps. First, the observers reviewed the 40 input images and their corresponding sets of 20 output images, selecting a few good diagnostic images from each set. The selected output images and the slope that produced them were recorded. The slope that produced the highest number of diagnostic images was 8 .

Second, the observers reviewed each input image and its output image as processed at mean intensity and with a slope of 8 , assigning a score of 1 (very low quality) 2 (low quality), 3 (good quality), 4 (very good quality), or 5 (excellent quality) to each input image and its corresponding single output image. The scores were recorded and analyzed.

The Student $t$ test was used to determine the significance of differences in mean score between the input and output images.

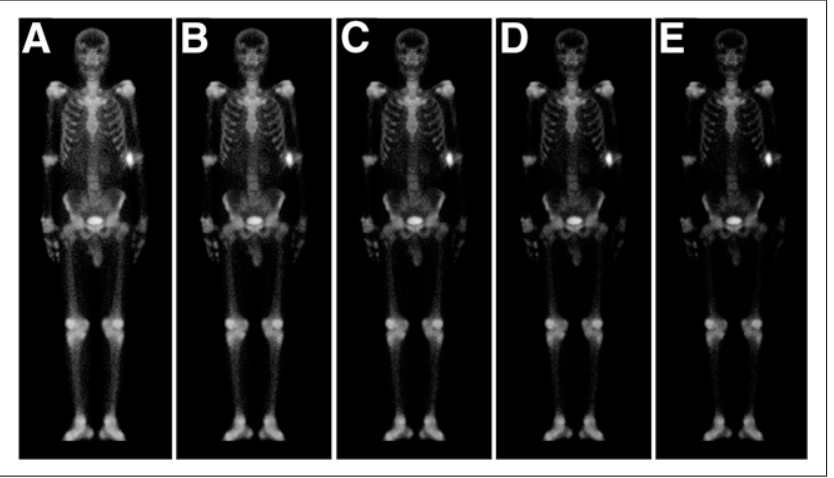

FIGURE 4. Processed whole-body ${ }^{99 m T c-M D P ~ b o n e ~ s c i n t i g r a p h y ~}$ images with slopes of 5-9 (A-E) and threshold equal to mean count in respective input image.

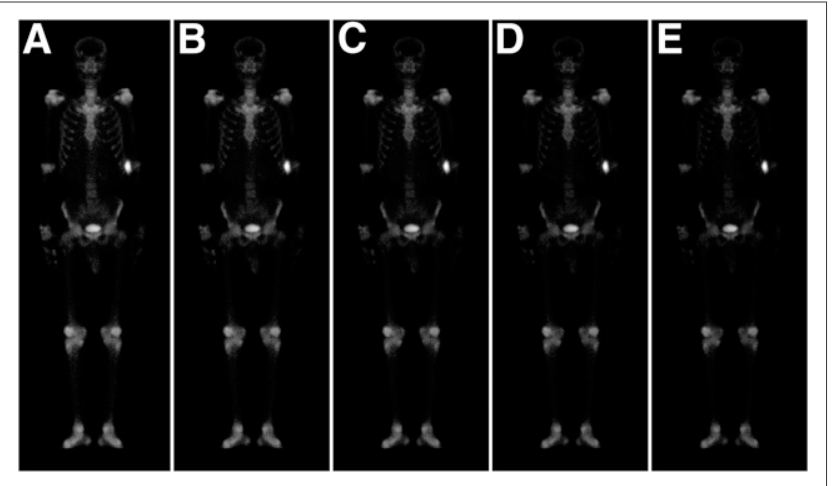

FIGURE 5. Processed whole-body ${ }^{99 m}$ Tc-MDP bone scintigraphy images with slopes of 10-14 (A-E) and threshold equal to mean count in respective input image.

The null hypothesis was that the IT function would provide output image quality approximately equal to input image quality at an $\alpha$ significance level of 0.05 .

\section{RESULTS}

Figures 3, 4, and 5 show example input images and their corresponding output images as processed at mean intensity and with slopes of $1-4,5-9$, and 10-14, respectively.

The number of times each slope produced clinically acceptable output images is given in Table 1 .

The IT function shifted all images to a higher quality score (Table 2), with 37 input images being scored below 4 and 33 output images being scored at 4 or above (Table 3 ). Hence, the quality of $89 \%$ of images (33/37) increased to a score above 3 .

The Student $t$ test was used to compare scores with and without use of the IT function applied, and a significant difference was found (mean score of 4.275 [variance, 0.861945] with IT function vs. 1.9375 [variance, 0.914187] without;

TABLE 1

Number of Times* Each Slope Produced Clinically Acceptable Output Images

\begin{tabular}{lr}
\hline Slope & $n$ \\
\hline 2 & 7 \\
3 & 20 \\
4 & 25 \\
5 & 20 \\
6 & 19 \\
7 & 28 \\
8 & 29 \\
9 & 18 \\
10 & 9 \\
11 & 4 \\
12 & 3 \\
13 & 1 \\
14 & 2 \\
\hline
\end{tabular}

${ }^{*}$ Of 40 total. 
TABLE 2

Score Assigned to Each of 40 Images Processed Using Slope 8

\begin{tabular}{|c|c|c|c|c|c|c|}
\hline \multirow[b]{2}{*}{$\begin{array}{c}\text { Image } \\
\text { no. }\end{array}$} & \multicolumn{3}{|c|}{ Observer 1} & \multicolumn{3}{|c|}{ Observer 2} \\
\hline & $\begin{array}{l}\text { Input } \\
\text { score }\end{array}$ & $\begin{array}{l}\text { Output } \\
\text { score }\end{array}$ & Difference & $\begin{array}{l}\text { Input } \\
\text { score }\end{array}$ & $\begin{array}{l}\text { Output } \\
\text { score }\end{array}$ & Difference \\
\hline 1 & 4 & 4 & 0 & 3 & 4 & 1 \\
\hline 2 & 3 & 5 & 2 & 2 & 5 & 3 \\
\hline 3 & 4 & 4 & 0 & 4 & 5 & 1 \\
\hline 4 & 3 & 5 & 2 & 4 & 5 & 1 \\
\hline 5 & 4 & 5 & 1 & 3 & 5 & 2 \\
\hline 6 & 1 & 5 & 4 & 1 & 4 & 3 \\
\hline 7 & 3 & 5 & 2 & 2 & 5 & 3 \\
\hline 8 & 1 & 4 & 3 & 1 & 3 & 2 \\
\hline 9 & 1 & 4 & 3 & 1 & 4 & 3 \\
\hline 10 & 3 & 4 & 1 & 2 & 4 & 2 \\
\hline 11 & 1 & 5 & 4 & 1 & 5 & 4 \\
\hline 12 & 3 & 4 & 1 & 2 & 4 & 2 \\
\hline 13 & 2 & 5 & 3 & 2 & 5 & 3 \\
\hline 14 & 2 & 4 & 2 & 1 & 4 & 3 \\
\hline 15 & 1 & 4 & 3 & 1 & 4 & 3 \\
\hline 16 & 2 & 5 & 3 & 2 & 5 & 3 \\
\hline 17 & 3 & 5 & 2 & 2 & 5 & 3 \\
\hline 18 & 3 & 5 & 2 & 3 & 5 & 2 \\
\hline 19 & 1 & 4 & 3 & 1 & 4 & 3 \\
\hline 20 & 1 & 5 & 4 & 1 & 5 & 4 \\
\hline 21 & 4 & 5 & 1 & 3 & 5 & 2 \\
\hline 22 & 2 & 3 & 1 & 2 & 4 & 2 \\
\hline 23 & 1 & 4 & 3 & 1 & 3 & 2 \\
\hline 24 & 3 & 5 & 2 & 2 & 5 & 3 \\
\hline 25 & 3 & 5 & 2 & 3 & 5 & 2 \\
\hline 26 & 2 & 5 & 3 & 2 & 5 & 3 \\
\hline 27 & 2 & 5 & 3 & 2 & 5 & 3 \\
\hline 28 & 2 & 5 & 3 & 2 & 5 & 3 \\
\hline 29 & 3 & 5 & 2 & 2 & 5 & 3 \\
\hline 30 & 3 & 5 & 2 & 2 & 5 & 3 \\
\hline 31 & 1 & 3 & 2 & 1 & 3 & 2 \\
\hline 32 & 1 & 3 & 2 & 1 & 3 & 2 \\
\hline 33 & 1 & 2 & 1 & 1 & 2 & 1 \\
\hline 34 & 1 & 2 & 1 & 1 & 2 & 1 \\
\hline 35 & 1 & 4 & 3 & 1 & 4 & 3 \\
\hline 36 & 2 & 4 & 2 & 2 & 5 & 3 \\
\hline 37 & 1 & 4 & 3 & 2 & 5 & 3 \\
\hline 38 & 1 & 3 & 2 & 1 & 3 & 2 \\
\hline 39 & 1 & 3 & 2 & 1 & 3 & 2 \\
\hline 40 & 1 & 4 & 3 & 2 & 5 & 3 \\
\hline
\end{tabular}

Difference $\geq 0$ indicates output image same as or better than input image.

$t(78)=11.7661, P<0.001$, confidence interval, -2.7330 to -1.9420 ; SD, 0.8884). This result suggests that the IT function does enhance bone scintigraphy images that exceed the dynamic range of the monitor.

TABLE 3

Number of Images Receiving Each Score

\begin{tabular}{cccccccc}
\hline & \multicolumn{3}{c}{ Input image } & & \multicolumn{3}{c}{ Output image } \\
\cline { 2 - 5 } \cline { 6 - 8 } Score & Observer & Observer & & Observer & Observer \\
& 1 & 2 & Average & 1 & 2 & Average \\
\hline 1 & 17 & 16 & 16.5 & & 0 & 0 & 0 \\
2 & 8 & 17 & 12.5 & & 2 & 2 & 2 \\
3 & 11 & 5 & 8 & & 5 & 6 & 5.5 \\
4 & 4 & 2 & 3 & & 14 & 10 & 12 \\
5 & 0 & 0 & 0 & & 19 & 22 & 20.5 \\
\hline
\end{tabular}
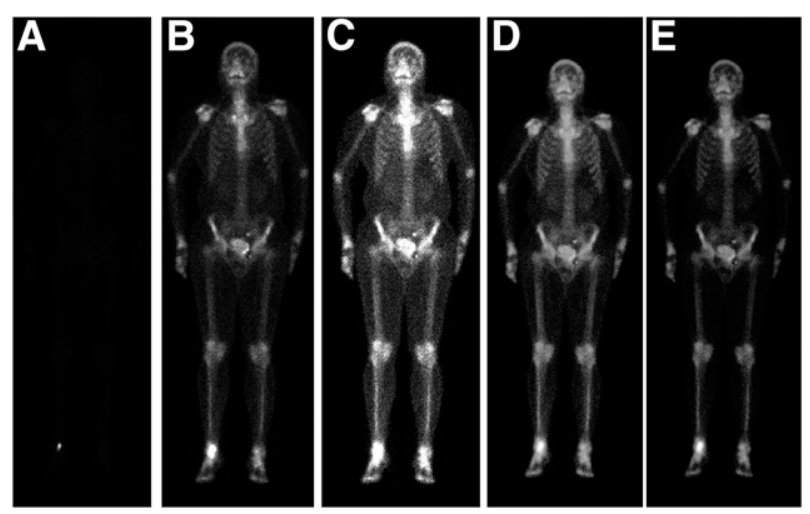

FIGURE 6. (A) Input image. (B and $\mathrm{C}$ ) Two images processed using window-level contrast-adjustment tool. (D and E) Two images processed using IT function.

Two images-one processed using the window-level contrast-adjustment tool (a digital imaging contrast-enhancement tool) and the other using the IT function-are shown in Figure 6. Relatively better image contrast was obtained using the IT function (Figs. 6D and 6E) than the window-level contrast-adjustment tool.

\section{DISCUSSION}

In this study, bone scintigraphy images exceeding the dynamic range of the monitor were processed using an IT function. The IT function enhanced the processed images, showing details that had not been visible in the dark and light regions of the unprocessed images. The observers determined that use of a slope ranging from 4 to 9 in the IT function produced images of acceptable quality. The largest number of acceptable images was produced when a slope of 8 was used.

Each slope renders a unique output image based on the shape of the curve. One common denominator is compression of the lower- and higher-intensity levels; therefore, a gray-level difference at these two ends may not be appreciable. In the case of bone scintigraphy images, compression of the higherintensity level may mask differences at this level that denote important clinical information. However, this type of information is also difficult to visualize with the existing, widely used window-level contrast-enhancement tool (Fig. 6).

Unlike the other output images, 2 images (Fig. 7) did not improve significantly at a slope of 8 , with their scores remaining at the lower end of the scale. One explanation may be poor uptake of the radiopharmaceutical, but this possibility cannot be confirmed because the study was retrospective. This result indicates that one image processing method may not fit all circumstances.

One limitation of the IT function is that a single value for slope may not improve all high-dynamic-range images; however, a range of slopes from 4 to 9 may improve most images. For instance, contrast needs to be improved in higherintensity regions such as near the bladder or near the extravasation site. But in such cases, the IT function may 


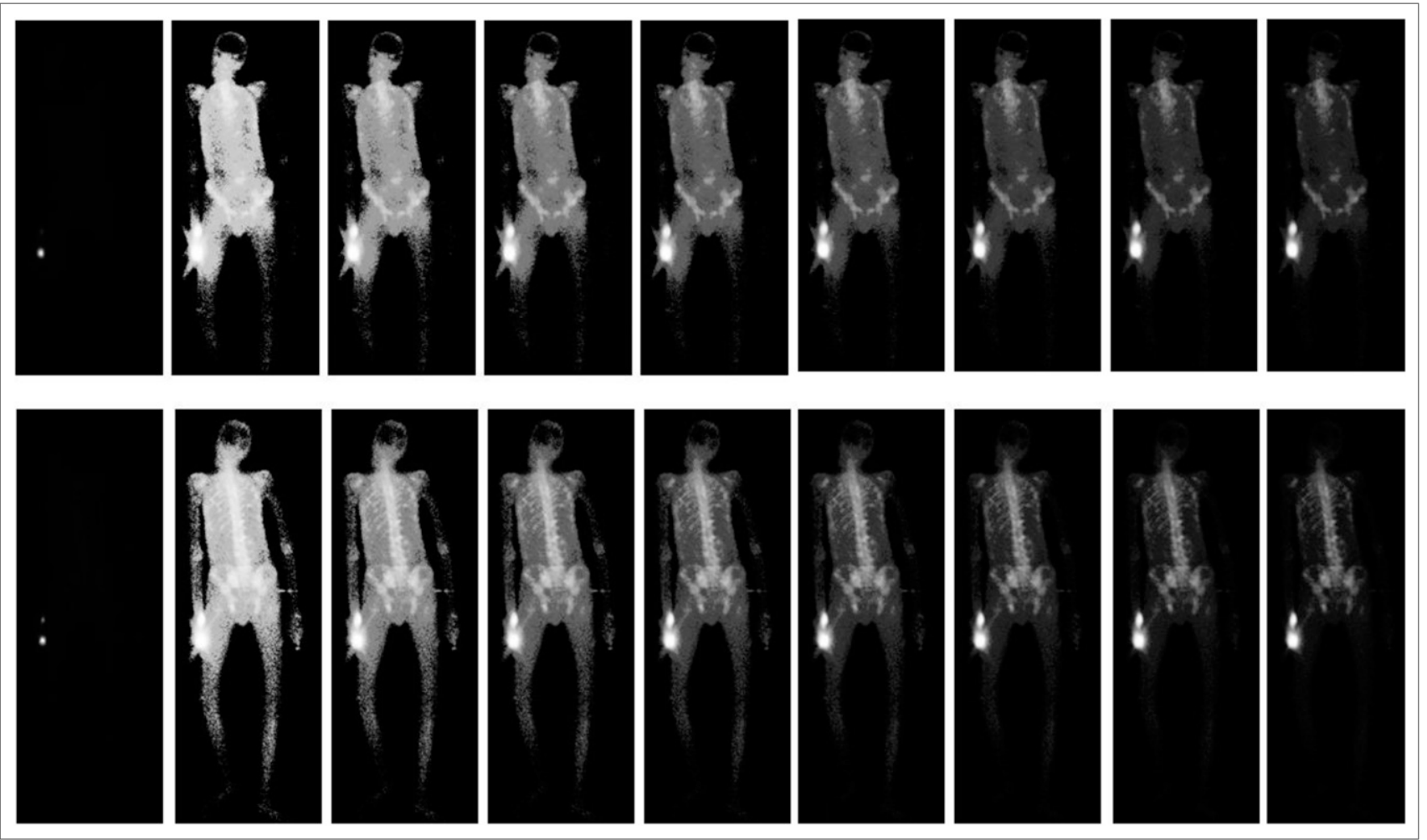

FIGURE 7. Two sets of whole-body 99mTc-MDP bone scintigraphy images that did not score on the higher end of the scale.

not provide a satisfactory result because it compresses the higher-intensity level in the image as well. Window-level contrast-enhancement tools are the most widely used method to adjust image contrast in nuclear medicine. These tools are interactive and driven by the familiar mouse pointer, but they require multiple trials while the nuclear medicine physician inspects the bone scintigraphy images to make a diagnosis. Moreover, even with multiple trials, the quality of processed bone scintigraphy images may often fall short. Many proven contrast-enhancement methods have been described in the literature, but their use in bone scintigraphy has not been investigated $(4,5)$.

Studies to improve the quality of bone scintigraphy images have been previously published (6-8). Verdenet et al. (6) used a global histogram equalization technique to enhance bone scintigraphy images. They found that the technique improves the readability of the images. However, they also observed a few false-positive results. Jeong et al. (7) compared 6 histogram equalization-based techniques and reported that the exact histogram specification technique gives the best performance. Ardenfors et al. (8) applied the Pixon algorithm (Siemens) to whole-body bone scintigraphy images acquired with half the standard scan time. They found that the processed images had sufficient clinical information but were of lower quality than images acquired with the full standard scan time.
The IT function is not specific for the type of image investigated in the present study but, rather, can be applied to improve contrast in any scintigraphy image. The extent of improvement may vary with the characteristics of the input image and the parameter values selected for the IT function. Our group has applied the same IT function to improve contrast in PET images of the liver and achieved positive results (9).

In the present study, contrast near the injection site and near the bladder did not improve as much as desired. We plan to investigate other image processing methods to address this issue. The practical finding of the present study is that, using the average intensity of the image as the threshold and a slope of 8 , the IT function improved about $90 \%$ of images exceeding the dynamic range of a monitor. Because each threshold and slope generate a unique output image, the present study also provides the guideline that a mean-intensity threshold and variable slopes of 4-9 should be applied in the IT function to get clinically acceptable bone scintigraphy images.

If the IT function were to be used by nuclear medicine physicians for certain low-quality bone scintigraphy images, repetition of the scan due to high dynamic contrast and poor visualization might be avoided. Moreover, the IT function has the advantage of being easily implemented using any computer programming language and any personal computer. In 
addition, monitors with the specifications used in this study are easily available and are more cost-effective than those provided by vendors in nuclear medicine setups.

\section{CONCLUSION}

High-dynamic-range bone scintigraphy images can be remarkably enhanced using the IT function.

\section{DISCLOSURE}

No potential conflict of interest relevant to this article was reported.

\section{REFERENCES}

1. Thrall JH, Ziessman HA. Skeletal scintigraphy. In: Nuclear Medicine: The Requisites. 4 ed. Philadelphia, PA: Elsevier Mosby; 2014:98-130.
2. Bergmann H. Nuclear medicine image display. In: Nuclear Medicine Physics: A Handbook for Teachers and Students. Vienna, Austria: International Atomic Energy Agency; 2014:512-546.

3. Pizer SM, Chan FH. Evaluation of the number of discernible levels produced by a display. Inf Process Med Imaging. 1980;88:561-580.

4. Gonzalez RC, Woods RE. Intensity transformations and spatial filtering. In Digital Image Processing. 3rd ed. New Delhi, India: Pearson Education; 2009:126-220.

5. Pratt WK. Image enhancement. In: Digital Image Processing: PIKS Scientific Inside. 3rd ed. New York, NY: Wiley; 2002:243-296.

6. Verdenet J, Cardot JC, Baud M, Chervet H, Duvernoy J, Bidet R. Scintigraphic image contrast-enhancement techniques: global and local area histogram equalization. Eur J Nucl Med. 1981;6:261-264.

7. Jeong CB, Kim KG, Kim TS, Kim SK. Comparison of image enhancement methods for the effective diagnosis in successive whole-body bone scans. J Digit Imaging. 2011;24:424-436.

8. Ardenfors O, Svanholm U, Jacobsson H, Sandqvist P, Grybäck P, Jonsson C. Reduced acquisition times in whole body bone scintigraphy using a noise-reducing Pixon-algorithm: a qualitative evaluation study. EJNMMI Res. 2015;5:48.

9. Pandey AK, Sharma SK, Agarwal KK, Sharma P, Bal C, Kumar R. Digital contrast enhancement of ${ }^{18}$ fluorine-fluorodeoxyglucose positron emission tomography images in hepatocellular carcinoma. Indian J Nucl Med. 2016;31:20-26. 